\title{
Olympia: la mirada femenina sobre los juegos olímpicos de Berlín
}

\author{
MaríaGracielaRodrígues*
}

\section{Resumo}

Em 1936, quando se celebraramos Jogos Olímpicos de Berlim, Hitler confere a tarefa defilmá-los a Leni Riefenstahl, uma espécie de documentariasta do teceiro Reich que já, em 1934, havia feito um sobre o $1^{\circ}$ Congresso Nazista. Olympiaé, de certo modo, ofilme oficial de Berlim 36, e, além de ser um documento da iconografia nazista foi realizado por uma mulher em um tempo onde isto não era comum na indústria cinematográfica.

O objetivo deste trabalho é fazer uma análise interpretativa sobre oolharfeminino que atravessa ofilme. Um olhar que, articulado com outros, secomplementaemumtodonarrativo indissociável doprimeiro.

A metodologia utilizadaéda análise cultural visto que permite desvelar os significados que aparecem na superficie textual de Olimpia, cujos sentidosalgunsmais explíccitos que outros-estãoplenos de referências contextuais.

Se Olympia se mostra como um vagaroso deleite comatletas masculinos e femininos desnudos, um tanto ousado para a época e para o gênero documental, esta ousadia nos remete a uma colocação no filme a um só tempo estético e ideológico somente possivel por tratar-se de umolharfeminino. Isto é o que este trabalho pretende demonstrar.

\section{Abstract}

In 1936, when the Olympics Gameswere celebrated at Berlin, Hitler put Leni Riefenstahl in charge of the film. She was a kind of an official documentary maker of the Third Reich and had already filmed
The Triumph of the Will, the filmabout the $1^{\text {st }}$. Nazi Congress in 1934. Olympia is, in a certain way, the official movie of Berlin '36 which is not only a document of the nazi iconography but a film made by a woman in times in which that was no usual in cinema industry.

The goal of this paper is to trace some lines of interpretative analysis about the feminine look that crossesthefim. Alookwhich, articulated tootherlooks, functions a complement of them. All the looks finally builds up a whole set in which the first is dissolved.

The methodology that I used is the cultural analysis in order to unfold the meanings that appear in the textual surface of Olympia. Thesemeanings-some more explicit than others- saturate the text of contextual references.

IfOlympiashows apleasantenjoying lookwhowatch naked female and male bodies, not common in those timesandin this genre, this gesture is addressed to both an aesthetic andideological collocation in thefilm whichisonlypossible because it is afeminine look. This is what this paper will try to demonstrate.

\section{Detrás de la mirada de Leni: un recorrido por el film Olympia}

“..., nudity played a large part in the representation of the masculine ideal type, as it had for Winckelmann earlier.Buthere suchnudity rarely referred to the Greek example; it referred nearly always to nature. The socalled turn-of-the-century rediscovery of the human body, as it has been called, was part of the search for the genuine as opposed to the artificiality of modern life, for unspoiled nature embattled againstmodernity."

GeorgeMosse 
Históricamente,lamiradasobreeldeportehasidouna miradaconstruidadesdeel puntode vistamasculino. Nosóloen las retóricas del espectáculo,endondela presenciafemeninaes exaltadadesde los atributosfísicosdelgénerosinotambiéndesdelasescasas aunque peculiares prácticas de algunasmujeresque,comoen loscasosdela 'GordaMatosas' y 'LaRaulito, , fueron objetode un registrocorporal (allídonde las propias prácticas masculinas se inscribían en cuerpos femeninosparaluegosernarradas por loshombres). Perotambiénenlos productosespecíficos sobreeltema deportivo, sean estos del género documental, periodísticooficcional,elenunciadorporexcelencia eselvarón.

Olympia,deLeniRiefenstahl,esunodelos pocoscasos en que esta relación se invierte y es la mujer-o acaso una mujer que condensa la mirada de las mujeres-laquenarraeluniversodeldeporte.Esto, si por un lado significauna fuerterupturaenel tipode imágenes que seconstruyen (masculinamente), no produce sinembargolainauguracióndeningún tipo de serieque abarque a productos culturales sobreel deportedesde unamiradafemenina. O,siacasoexisten, noparecen poseerni lamasividad ni larepercusión históricay simbólicade Olympia.

Loque ocumeesqueLeniRiefenstahlnosólomiraun eventodeportivoy cultural paradevolverlomundializadoenel circuito que inaugurabael cine, sinoque tambiénestámirandoun suceso políticodeconsidera-bles dimensiones. Dehecholos Juegos Olímpicos (JJOO) de Berlínfueronobjetode agudasinterpretacionesqueterminaronconvirtiéndolosenunaespecie de símboloque condensa todos los repertorios de la 'utilización política del deporte'. Y sobreeste hecho políticoLeni Riefenstahl produce unamiradaestética eideológicar que podríarelacionarse con el contexto de producción del momento pero también con su condicióndegénero.

Aunquelarelacióndelaestéticacon lopolíticoesmuy complejay los debatesentomoaestarelaciónrecomen tantolasdiscusionessobrelaautonomíadelartecomo loscontextos deproducción del artistaen sí,noes mi intención discutiraquílos 'usos' propagandísticosde los productos massmediáticos. El vínculodelEstado conlos medios decomunicación yelescenariopolíticoesunarelación de complementariedad antes que deutilizacióndealgunoporotro.ComoafirmanMcFee yTomlinson, toda vezqueunEstado, conun sistema deportivomedianamenteorganizado,formulasus objetivos propagandísticos, esfácilimaginarcómoesos objetivos pueden lograrse usando los medios de comunicación(McFeeyTomlinson, 1995).

Lociertoesque, comounanuevacapadesentido, ala estilización del hechopolítico sele superponen otras dos dimensionesculturalesque sesganel análisismeramente ideológico: por un ladoel marco cognitivo que conceptualiza la gimnasia de una particular maneraenunespecíficomomentohistóricoy porel otro la mirada femenina, produciendo registros visuales-acaso 'involuntarios'-de cuerpos embellecidos, fundamentalmente masculinos perotambién femeninos.

Yeséstaunaclaveparaentenderlaestéticade Olympia: sólolosojos deunamujerpodían sortearlacertezade una condena social que hubiera visto en su película untratamientohomosexual.Los ojos permisivos de Leni Riefenstahl traducen su goceen detallistastomas decuerposmasculinos, inclusoeldelmismísimoJesse Owens, velocista negro que desafió la supuesta superioridad aria. Allí se detiene -y se deleita-la Riefenstahl.

Enlasuperficie textual de Olympia, saturadade sentidos, algunosmás explícitos que otros-velados, desvelados orevelados-esmi intención señalar, aunque sóloalosefectosdelanálisis, tresmiradassuperpuestas: a) lamiradaalemana, colocadasobre una Greciaidealizada, naturalizaday, porende, ideologizadaquees lamiradaque anudael finideológicoperseguido: la de mostrar los atributos de unaraza que se pretende perfecta; b) lamirada 'conceptual' que, al discriminar entre gimnasia y atletismo de los deportes de situación, ${ }^{3}$ focaliza la atención en la corporalidad, 
estetizandoa los dos primeros en detrimento de los otros; c)lamiradafemenina, posadasobreloscuerpos, que expresala fruición de una visión desenfadaday hasta perturbadora y que, fundamentalmente, goza: Olympiaesunmorosodeleitarse con atléticoscuerpos femeninos ymasculinos, acasoosados paralaépocay parael génerodocumental.

Másquelos símbolos abstractoscomobanderas, monumentos o inclusive esculturas, la utilización de cuerpos humanos vivientes, proporcionóal régimen un modo de auto-representación. "Estos cuerpos broncíneos",afirmaGeorgeMosse, "seconvirtieronen unsímboloabstractodelabellezaaria, nomenosque los atletasdelfilm sobrelos Juegos Olímpicosde 1936 deLeniRiefenstahll'(1998: 173).

Laintención de este trabajo es trazar algunas líneas de análisis sobre cada una de las miradas señaladas que, alavezque son discernibles en la textualidadde la película, se complementan en un todo narrativo indisoluble.

\section{La triple mirada de Olympia}

En1936,cuandosecelebran los Juegos Olímpicos de Berlín,HitlerencomiendalatareadefilmarlosaLeni Riefenstahl,especiededocumentalistaoficial del Tercer Reich quien ya en 1934 habíafilmado El triunfo de la voluntad, la película sobre l ler. Congreso Nazi. Olympia es, de algún modo, el filmoficial de Berlín '36, el cual, además de ser un documento de la iconografía nazi, fue realizado por una mujer en tiempos en que esto no era corriente en laindustria delcine.

Olympia (Juegos Olímpicos de Berlín 1936o Los dioses del estadio) estácompuestadedospartes: ‘La fiestadelabelleza» (115 minutos)y «Lafiestadelos pueblos»(89minutos). Tantolaconcepciónyladirección como el montaje estuvieron a cargo de Leni Riefenstahl.Lafotografíaes deHansErltylos Juegos fueron cubiertos por 34 camarógrafos. Losautores de lamúsicason WalterGronostayyHerbertWindt.
Se ha dicho que Olympia es una película paradigmáticaenlacualelartedesafíaalopolítico.Enverdad, la dificultad de analizar el film reside en que las significaciones socialesque devienen del propiocontexto histórico (el Berlín del Tercer Reich) tiñen cualquier otra aproximación que no seala política. Sin embargo, si por el otro lado, solamente nos detenemosa observar su construcción estética, seguramenteperderemosde vistalascomplejas relaciones entrelas retóricas, laconstrucciónideológicay los discursos de las disciplinas corporales que informan al film.ComoafimanMcFeeyTomlinson: "Aliadoalas innovacionesartísticas, el potencial del textode hablar paralaeternidadtieneelefectomistificantedeelevarse porsobrelasespecificidadessocio-históricasysobrela colocaciónpolítica"(1995:230).

Porotrolado, eselcuerpomasculinoelquepredominaen estacolocación política, en función delaposiblidadderepresentación de una camaradería, esencialmentemasculina. Contrariamente, eldesnudofemenino,comoafirmaMosse, nopermite suinclusiónen clavedeejerciciodefunciones públicas ${ }^{4}$.

Para dar cuenta de estas relaciones complejas, la propuesta es recorreranalíticamentelas tresmiradas mencionadas que se superponen en el film, a fin de revelar el contenido ideológico no tanto desde la remanida situación políticaespecíficasino, más bien, desde la trama que vinculalas formas estéticas de la cultura corporal y la celebración de la fraternidad masculina comoun auténtico y profundo sentidofilosófico.

a) Lamiradaalemana: la operación ideológicade Olympia aparece en torno a la construcción del nacionalismo y del culto a una raza. El film comienzacon planos decuerpos 'silvestres' ydela ciudaddeOlympiaenruinasdondeseenciendeuna antorchaque recorreráel trayecto de Olympiaa Berlín, sobre el fundido de un mapa de Europa. Acasodesdeel puntode vistadellenguajecinematográfico, puede considerarse esta construcción significantecomoun sobretrazooquizáscomouna 
operación de didactismo sobre un público recientemente ingresadoen el circuitofílmicoy cuyas competencias cinematográficas estaban siendoobjetode unaprendizajecultural.

Laantorchaolímpica, símboloideadoporCarlDiem paralos JJOOde 1932, y sutraspaso, relevohistórico cuyoritual seinauguraen Berlín, ${ }^{5}$ se acomoda con toda su carga simbólicaen el escenario olímpicoy se convierte en el hilo conductor del pasaje de formas entrelas ruinas delaciudad deOlympia, endondelas estatuas cobran vida, realizan movimientos gimnásticosy atléticos yluegoenciendenlaantorcha, yel urbanoy cosmopolita Berlín, que quedade este modo investido con los valores clásicos de la antiguiedad. Enel trayecto, los atletas seirán vistiendo y deberánatravesarlugaresurbanizados, dejandoatrás el jardín del edén de Olympia. Asistimos a la construccióndel 'aura' ensumáspuraybenjaminiana definición: "La manifestación irrepetible de una lejanía”(Benjamin,1973).Enestaespeciedemontaje conceptual las imágenes nos han llevadode la pura naturaleza a la civilización, de los íconos inconfundibles de la antigua ciudad derruida a los nombresdelasmodemas ciudades superpuestoscon respectivas tomas áreas, de lacarrera de un solitario atleta por unaplayadesiertaa la travesía de unalínea dibujada enel mapadeEuropa, guiando al receptor, instruyéndolo.

Eldesnudomasculinocomoun símbolonazi, afirma Mosse(1988),es deinspiración griega: el ideal ario,en comparación conelantiguotipoideal griego,permite integrar lasideas de saludmental y corporal.Según la línea de pensamiento que sostiene al ideal ario, éste penetróalNortedeEuropadesdeGrecia, pasandoporla India y, en esta trayectoria tomó lo mejor de la civilizacióngriega. Sinembargo, advierteMosse, elnudismo,enestecontexto,nodebeconsiderarsecomouna prácticaaltemativistasinocomoelredescubrimientodel cuerpohumanocomofuentedenaturalezaybelleza.

En otro sentido, tambiénhay queentenderel reenvío ala Antigua Grecia como producto de un encanta- mientocon lacultura griega profundamente arraigadoentrelos intelectualesalemanes. Yaamediadosdel siglopasado, Olympia, la mítica ciudad griega, era reivindicada por los alemanes como un lugar sentimentaly particularmente suyo. 'Eraterritorio sagradoenunmundogobemadoporotros diosesquefueron capaces deimponer lapaz gracias a latreguaolímpicapero sigue siendoterritorio sagrado paranosotros también. Ese hechizo, ese amor por la patria, la consagración al arte y el empeño puesto en la consecucióndelafelicidad, todas esascosas deben ser de nuevo ofrecidas a los límpidos rayos de nuestro mundoilustrado"(Mandell, 1990:33), declarabaen 1852EmstCurtius, unintelectual alemánqueademás dedesempeñarsecomoprofesoren Berlín, eratutor del príncipeherederodePrusia. «Allí,yaciendoenlo profundo, se encuentra la vida de nuestras vidas», enfatizabaCurtius. Y sus ruegos tuvieron eco: entre 1875y 1881 Alemaniarealizó6expedicionesaGrecia paradesenterrar las ruinas de laciudad olímpica, que culminaron con la creación de un museolevantado porlosalemanescercadeOlympiadondeestánlosfrisosdeltemplodeZeus.

DeallíquelosJJOOdeBerlín’36, significaranlaúnica perooportunaaparición públicadeSpiridonLoues,el griegoganadoren 1896delaprimeramaratónmodema. Loues, quiennuncamás volvióacompetirenunencuentro intemacional, desfilóencabezandoladelegacióngriega enlaceremoniadeinauguracióndelos JuegosdeBerlíny luegolehizoentregaaAdolfHitlerdeunaramadeolivo, símbolodelapaz, procedente del bosque sagrado de Olympia.Laempresahabíarendidosusfrutos.

b) lamirada 'conceptual': elénfasisenlobucólico(característicaque yase habíamanifestadoen ElTriunfo de la Voluntad de 1934)y en laestética delo corporal,dejapocoespacioal 'guión' delaceremonia olímpica, quecasinoes registrada por los ojos de Leni Riefenstahlmásfascinados porloscuerposy porelmovimiento. Dehecholabanderaconloscincoanillos $^{6}$, oeldesfile de delegaciones sonapenas signosdepuntuaciónenel gran relatofilmico. 
Leni Riefenstahlmásbiensedesvíapresentandoeventos no pautados o tomas donde algunos encuadres hacen sospechardela veracidad de algunas escenas deportivas ${ }^{7}$.Ejemplodeloprimeroeslaexhibición masivadegimnasia-fueradel programaolímpico-en unasecuenciadondeelmontajecomienzaconplanos que toman lacercaníaindividual de un torsoy se va ampliandohastallegaraunapanorámicademiles de gimnastas sincronizadas en un mismo y repetitivo movimiento. ${ }^{8}$ Ejemplos de los encuadres a primera vistaapócrifos, sonalgunas tomas comolasubjetiva del corredor de maratón y algunos close-ups de gimnastasque, de serciertas, hubieran dificultado su performance, teniendo en cuentaqueel desarrollo tecnológicodelmomentonohacíaposiblelaportación demini-cámaras porparte de los atletas.

Luego, más adelante en el relato, Leni Riefenstahl disfrutará-yharádisfrutaralosespectadores-conuna secuenciade saltos omamentales armadacon planos encontrapicadaque, enunacomplejidad estéticaextrema, editará alternando con peculiares tomas en reversa. Esto produce un efecto de extrañamiento primeroy defascinacióndespuésqueinterpelaauna recepciónlaboriosa, obligando al receptoraunfuerte trabajodedeconstrucción.

Porsuparte,losdeportes situacionales(fútbol,hóckey, etc.)sonelobjetodeunascámarasmuchomenoscreativas ymáscentradaseneleventoensí.O, sielanacronismo funciona, más 'televisivas', casianticipatorias delasgramáticasaudiovisualesdeportivascontemporáneas.

PareceríaquelamiradadeLeniRiefenstahl-femenina perotambiénalemana-estáexpresandoladefensadel 'tumen'-dedondeluegoderivaránlagimnasiadeportiva yotrasdisciplinas-frentealavertientedelos 'sports' preferidapor los anglosajones. Dehecho, es sabidoque, cuandoen 1896elBarónPienredeCoubertinrestauralos JuegosOlímpicos,Alemaniaseoponeaparticipardela propuesta:los 'sports' ingleses, llamados aserlos protagonistasdeldeportemodemo,aparecíancomoelenemigo númerounodel 'turnen', sistemadegimnasianacional alemánrecientementecreadoporFriedrichJahn, quien
oportunamentehabíadeclarado:"Losdepoitesdestruyen loqueelTumensehadedicadoacrear:unacomunidad nacional'"(citadoen Guttmann, 1994: 144).

EsterechazodeAlemaniaaparticiparenlosprimeros JJOOmodemos pudodeberse, en parte, alahistórica relacióndeenemistadcon losfrancesesperoenmayor medidaporqueveíanlaintemacionalizacióndeportiva comounenfrentamientoalos sistemasnacionales de gimnasia, entreloscualesel 'turnen' alemán gozóde gran difusión en algunos países comola Argentinaentre otros - a través de clubes que replicaban en lo localelmétodoy los principiosquelo sustentaban?.

Demodoque Olympia también permite verlascontradiccionesqueexistánenelcampodeldeporteenese momento.Porunlado,sienlapelículaaparecenlos 'sports', habráqueleerlocomolaaceptaciónde uninternacionalismoyaprácticamenteinevitable.Porelotrolado,el 'turnen' convivíaen Alemaniaconalgunas prácticas deportivasanglosajonasaunqueenverdaden posiciónde subaltemidadrespectodelprimero(Mandell,1986).

Porúltimo,lamiradadeLeniRiefenstahlsedetiene, sinculpas ni necesidaddenegociar, en los productos derivados del 'turnen' y no se ocupa de colocar la convivenciaconlos 'sports' enclaveconflictiva. Esto nicae en lalógicadel relatonitampocoesimaginable enesemomentodeladifusión deportiva. Quizássilos JJOOde Berlín hubieran sucedidoen 1916, como habían sido programados en un principio antes de estallarlaPrimera GuemaMundial,lasdiscursividades en este sentido habrían diferido. No es sino hasta cuarentaaños despuésdelarestauracióndelos Juegos Olímpicosmodemosque Alemanialograhacerlosen su casa. Berlín sería entonces la oportunidad para mostraral mundoel vínculodel míticopasado olímpicoconlos postuladosideológicos del régimennazi. Perolaluchacontralos 'sports' yaestabacasiperdida.

c) lamiradafemenina:esenestadirección-yaquíreside lafortalezadelanarratividadde Olympia-queelrelatodeLeniRiefenstahlseregodeaenlasignificación que la tercera de las miradas le otorga: el de su sensibilidadfemenina. Yentoncesloideológicotermi- 
nadecobrarsentido.Lapresentaciónyre-presentación delpoderdelafratemidadmasculina, requeríauna miradaparticular,quepudieradeleitarseobservando cuerposmasculinosenuncomunitariosaunasinser acusadadehomosexual.ComoafimaGeorgeMosse: "El cuerpo masculino todavíaejemplificabala armoníaylavirilidad, ahoraenmarcadaporlossímbolosdelogenuinoyloetemo".(1998:97).

Aunqueel usodel cuerpodesnudoseremontaenel nazismo, como expusiéramos previamente, a la tradición griega, estautilización "presentabaun problemaespecial parael Nacional-Socialismo porque, ¿cómo podía reconciliarse esa desnudez con la respectabilidad, tan importante para eléxito del movimientopolítico?'(Mosse, 1998: 172).El análisis deltextopermiteafirmarqueparaLeniRiefenstahl(y conellaparaelideariodelrégimen)elcuerpoatlético sobrepasalos límitesdelopolítico.El tratamientode locorporal enel film puede considerarse comoparte deunmecanismoretóricoquelograinvestirdeunsentido filósoficoaloscuerpos de la fraternidad masculinaalemana Yaunqueel sujetosocial siempredesborda al sujetoimplicadoporel texto, aquélyéstepertenecen almismodominiodiscursivo.Enestesentido, elefecto producidoporestaresemantizaciónnodebesertomadocomoel resultadodeunaduplicacióndelossujetos empíricos sinocomoel posicionamientoentresujetos y textos, producidos por el propio discurso que los atraviesa (Morley, 1996), en este caso el de la superioridad delarazaaria. Deallíquelamiradaque seposasobreloscuerposalemanesdistedeserlamisma deaquellaque simplementeechaun vistazoalos atletas (nuncadesnudos) de otras nacionalidades.

El cuerpo-el cuerpo alemán-desde esta peculiar mirada, setransformaentoncesenun símboloa-históricoque,por laremisión aun pasadonaturaly, por lotanto,inapelable, merecesercelebrado.Nisiquiera ser objeto de propaganda, sino de celebración y regocijo.Deestemodo Olympia, adiferenciadeotros filmesdocumentales, re-elaboray escenificaunsimbolismoqueseubicaporencimadelospropios JJOO de Berlínque son sureferente: su preocupaciónnoes tanto narrar los sucesos de los JJOO sino más bien colocarloscomoel fondode otrafiguraen relieve.

Lociertoesqueelefectodesentidoqueubicaalocorporal más alládela historia, nolaproduceun miembro de lapropiafraternidad masculinasinoquellevapor trazo la mirada de una mujer. Yesta mirada puede considerarse comounacoartadadel régimentodavez queotrosojosnohubieranpodidoescenificaryconvalidartaneficazmenteunimaginariosocial pre-existente. Deestemodopuedeafirmarse,conMcFeeyTomlinson (1995) que Olympia es un testimonio a futuro de lo que unatradiciónbasadaenelelitismofísico, pondría enjuegotrágicamente duranteelHolocausto.

\section{Conclusión}

Las tres miradasseñaladas, al superponerse, producen unefectode naturalización y de constitución de una filosofía que subyace al idearionazi. Demodoque Olympianopuedeconsiderarseespecíficamentecomo unapropagandasinocomountestimoniodeaquél.De hecho, comoseñalaHart-Davis (1986), lasescenas dedicadasaJesseOwens apuntanenunadireccióncontrariaaloque seríaunapropagandadetipopolítico.

Dichoen otras palabras,Leninodescubrelapólvora cuandose internaen laforestapersiguiendoaese grupodehombresy luegoseinstalajuntoconellosenel saunaaobservarlosdesnudos frotándoseconaceites. Enciertomodo, estos actosyaestabaninscriptosenel recorridodelamemoriahistórica: desdelamismísima Atenas hasta el 'turnen' y de allía 'la bellezade los cuerpos', títulodelsegundofilmde Olympiaenelcual aparecen lasmencionadasescenas, hay unalínearecta.

Sóloque unamiradamasculina, en 1936, no hubiera podido poner en juego la sensualidad que exuda Olympia.PeroLeni, aquien Hitlerledio34 cámarasy amplia libertad paraeditar, no sólopudohacerlosin restricciones sinoque disfrutódeello. El costo, claro, fuepasar4 añosen un campodeprisioneros francés luego de la caída del régimen y laimposibilidad de volverafilmar.Peroesaes otrahistoria. 


\section{Referências}

BENJAMIN, W.Elarteenlaeradesureproduccióntécnica.In: Discursos interrumpidos I. Filosofia del arte yde la historia, Madrid,Taurus, 1973.

GUTTMANN,A.Games \&Empires.ModemSportsandCultural Imperialism. NovaIorque, ColumbiaUniversity, 1994.

HART-DAVIS,D.HitlerGames. NovaIorque,Harper\&Row, NuevaYork, 1986.

MANDELL, R. Historia Cultural delDeporte, Barcelona, Bellaterra, 1986.

MANDELL,R.Lasprimeras Olimpíadasmodernas.Atenas 1896, Barcelona, Bellaterra, 1990.

MCFEE,G:;TOMLINSON,A.Notesonbodilycultureandsport Ideology: the case of Leni Riefenstahl, In Sport Culture Tradition,Actas delColoquiohomónimo, ComitéRegional de laUniónEuropea, 14 al 16 de mayo de 1993 (pp. 230-234), 1995.

MORLEY,D. Televisión, audienciasy estudios culturales. Buenos Aires,Amorrortu, 1996.

MOSSE, G. The image of man. The creation of modern masculinity.New York, 1998.

TARRUELLA,R.Docykistch(losojosdeLeni).In:ElAmante, no 13, marzo, 1993.

QUINTÍN Las vidas de Fräulein Leni, In: ElAmante, $n^{\circ} 13$, marzo, 1993.

*Institutode Investigaciones "Gino Germani”, Facultad de CienciasSociales(U.B.A.).Proyecto.ProyectoUBACyTSO01, 2001-2002:"Fútbol y aguante: imaginariomasculinoy cuerpo popular".'Director:PabloAlabarces.

\section{Notas}

${ }^{1}$ La 'GordaMatosas' fue unafamosahinchadelclubRiverPlate duranteladécadadel'40, quenose caracterizabaprecisamente porsufemineidad.LaRaulitoeselapododeunahinchadeBoca Juniors, una mujerque harenunciado a su género; entre otras cosas,porqueeramenoscomplejosobrevivirbajoestacondición comoniñadelacalle

${ }^{2}$ A pesar de que la cineasta siempre se consideró a símisma comoa-política y partidaria de un mundobucólico y menos contaminadoporlacivilización. Aún más, cadavezquerecibía acusaciones acerca de su apoyoal régimen nazi, nodejaba de sorprenderse. Para ampliar ver Tarruella (1993) y Quintín (1993)

${ }^{3}$ Se denomina deporte de situación a aquellos deportes que requieren la aplicación veloz de respuestas motrices únicas, irrepetibles, segúnel contextosituacional puestoenjuego.Los deportes de situación notienen movimientos queempieceny terminenen símismos, sinoque cadaunodeellos se elaboray ejecutadeunasolavezy parasiempre, enfuncióndelas relacionesespacio-témporo-objetales de lasituación. Estolos hace radicalmentediferentesdelos deportes cíclicos, quesonaquéllos que repiten sus gestos invariablemente yaque su contextono ofrecemodificaciones significativas de situación, comosonlos casos del atletismo, la natación, lagimnasia deportiva, etc.

${ }^{4}$ AfirmaMosseque “(E)ldesnudofemeninocomosímbolode labellezaariafemenina, entodocasonopresentabatratamientos inmediatos de respetabilidad porque, al contrario que los hombres, la mujer no desarrollaba funciones públicas de imporanciani en el Partidoni en el gobiernodel Tercer Reich. La mujer simbolizaba a través de su pureza y su castidad, la normativaideal delafemineidad"'(1998: 177).

${ }^{5}$ EnelfilmdeLeniRiefenstahlaparecentradicionesolímpicasque sonrecreacionesdesviadasdesusprecedentesculturalesyotras inventadas' paralaocasión, comolacampanaolímpicaquenunca másvolvióaseroídaenlosJJOO.

${ }^{6}$ LabanderaolímpicafuecreadaporelBarónPierredeCoubertin en 1913, sóloque por lainterrupción bélicarecién se presenta ensociedadenlosJJOOdeAmsterdamde 1928.

${ }^{7}$ Aunqueesnecesariodestacartambién, ensufavor,lafilmación, sincortesni interrupciones de lacarrerade 1500 metros.

${ }^{8}$ Muy similaraloquese vio, cuarentay dos años después, enel MundialdeFútbolde Argentina 1978.

${ }^{9}$ En Argentinael SistemaNacional deEducaciónFísica, elaboradoporE. RomeroBrestenocasióndelareformapropuestaen 1898 porelentonces ministrode InstrucciónPública, Dr.Luis Beláustegui,formaparte deestemovimientodifusionista, apesardequeel Sistema, antes que unaréplica, debe considerarse más biencomounaadaptación del 'turnen', de cortenacionalista.

Recebido:07/11/02

Aceito:10/12/02 
